of the most important European and American species is summarized. To the British reader it is regrettable that that of Münch, Rübner and others on the European larch is not included, but a selection had to be made and, for American readers, this species is of minor importance.

Though the artificial treatment of seeds and the requirements of artificial regeneration receive so much attention, the author rightly stresses the fact that the problems of natural regeneration are also bound up with seed production, storage, dispersal and germination, as well as the influence of biotic, climatic and soil factors on the survival of seeds in a viable condition and on the establishment of the seedlings as independent organisms. All these matters receive consideration in this work. The method of the author is to devote a chapter to each branch of the subject, giving a general account of the points investigated by different workers, with a summary of the most important contributions. Then a general review of the position is given, indicating points on which agreement is reached and those in which further investigation is called-for. A very full bibliography is added to each chapter. At the end of the book is a useful glossary of seed terms with their French, German, Danish, Norwegian and Swedish equivalents, and a list of authors mentioned in the text.

The work will be indispensable to research workers in the field of tree seed and very useful to practical foresters and members of the seed trade. It is not a handbook of tree seed, giving cut and dried information about individual species, but a reference book and a guide, not only to existing knowledge but also to the directions in which that knowledge should be extended in the future.

T. Thomson.

\section{NON-EUCLIDEAN GEOMETRY}

\section{Non-Euclidean Geometry}

By Prof. H. S. M. Coxeter. (Mathematical Expositions, No. 2.) Pp. $x v+281$. (Toronto: University of Toronto Press, 1942.) 3.25 dollars.

$\mathrm{T}$ HE philosopher Kant declared that Euclidean geometry was inherent in the human mind and expressed the truth about space. We now recognize that non-Euclidean geometry is equally valid as an abstract system, and that one particular form (due to Riemann) has more claim than Euclidean geometry to represent the properties of physical space. The transition from the old point of view to the new has revealed the true nature of geometry, and thence of mathematics in general, and has helped to build up the theory of relativity.

To survey their fields and build the pyramids, the Egyptians had a set of empirical rules, which the Greeks developed into a science. In particular, Euclid attempted to deduce the properties of "straight lines and circles from certain definitions, axioms, and postulates. The axioms or "common notions" were regarded as self-evident truths, whereas the postulates were frankly assumptions, but most of them seemed so obvious that no one denied them. There was one exception, namely, the fifth postulate (often called the eleventh axiom): If a straight line falling on two straight lines make the interior angles on the same side less than two right angles, the two straight lines, if produced indefinitely, meet on that side on which are the angles less than two right angles. For centuries mathematicians, while not doubting the truth of this postulate, objected to it as too complicated. It was hoped to prove it by showing that a contradiction would arise from denying it or one of its simpler substitutes, such as 'Playfair's axiom': Two intersecting straight lines cannot both be parallel to the same straight line. No contradiction was ever found. In 1823 Bolyai, and independently Lobatschewsky in 1826 , worked out a system of geometry (now known as hyperbolic) quite as logical as Euclid's, but assuming that his parallel postulate was untrue! Similar results had been obtained by Gauss even earlier, but for fear of ridicule he did not publish them.

A much greater departure from the Euclidean geometry appeared in 1854, when Riemann, using the methods of differential geometry which Gauss had developed from land-surveying, introduced a geometry (now called spherical) in which there are no parallels, and two straight lines enclose a. space! The relationship between the alternative geometries remained obscure until much later. Cayley (1859) showed that the 'metrical' properties of Euclidean space, such as distance and perpendicularity, could be derived analytically from the 'projective' (that is, non-metrical) properties of a certain conic called the "absolute". Klein (1871) developed this idea, and set up a general projective geometry, from which he obtained elliptic geometry (akin to Riemann's), parabolic (Euclidean), and hyperbolic. He also showed the existence of 'models' or correspondences, by which it could be deduced that one geometry is logically self-consistent if another is so. (Dr. Coxeter thinks that the only ultimate test of consistency is based on the properties of the physical world as interpreted by our senses.) More searching investigations into the logical structure of geometry were made by Pasch (1882) and Hilbert (1901). The word "axiom" is now used for an assumption concerning the relationship between certain undefined terms such as point and straight line, and there are an infinite number of geometries corresponding to the infinite choice of the set of axioms, which may be freely chosen as long as they are self-consistent. Naturally, those which agree closely with the properties of physical objects are of most interest, in particular Euclidean geometry because of its simplicity, and Riemannian because of its use in relativity.

Dr. Coxeter's book starts with an excellent introductory chapter, mainly historical. Chapters 2 and 3 are on projective geometry, and instead of defining polarity with reference to a conic, follow von Staudt by defining polarity as "a correlation of period two" and then look for a corresponding conic. Chapter 4 is on homogeneous co-ordinates. After these somewhat prolonged preliminaries, elliptic geometry is obtained from projective geometry, since every axiom of the former is valid in the latter. This idea is developed in Chapters 5, 6 and 7. In Chapters 8 and 9, Euclidean and hyperbolic geometry are derived from a general 'descriptive' geometry. The remaining five chapters are simpler, dealing with hyperbolic geometry in two dimensions, circles and triangles, a general triangle of reference, area, and Euclidean models. The book concludes with a bibliography and an index. It will appeal to those who have already a first acquaintance with the subject and are ripe for a systematic treatment. 